\title{
Erratum: Causal electric charge diffusion and balance functions in relativistic heavy-ion collisions [Phys. Rev. C 97, 014906 (2018)]
}

\author{
Joseph I. Kapusta and Christopher Plumberg (1) \\ (Received 17 April 2020; published 1 July 2020)
}

DOI: 10.1103/PhysRevC.102.019901

In the process of validating numerical results and preparing a paper [1], we discovered a coding error in our implementation of Eqs. (46) and (47) of our original paper. This affected several of our results for colored noise, those with finite $v_{Q}^{2}$ or $\tau_{Q}>0$. Although the error does not affect our most important conclusions, several of the figures from that paper are altered at the quantitative level. For completeness, all figures are shown below along with a few brief comments on the implications.

The self-correlations shown in Fig. 1, and the balance functions shown in Fig. 5, whereas their normalizations differ somewhat from those of the original paper, are virtually unaffected. The $k$ dependence of the density-density correlator (with self-correlations removed) remains largely unaffected, although some nonmonotonicity is observed in the $k=0$ intercept of the correlator with increasing $\tau$ in the uppermost panel where $v_{Q}^{2}=1 / 3$ (see Fig. 2). The most significant changes occur in the regular part of the self-correlations in rapidity space $\Delta \xi$ after only $5 \%$ of the total evolution has elapsed. Here, in contrast with Fig. 3 of our paper, we now find only a single set of wavefronts reflecting the causal propagation of correlations in rapidity separation. The single set of wavefronts still occurs at $\Delta \xi=2 \xi_{s}$ as shown in the upper portion of Fig. 4 in the original paper, whereas the set of wavefronts at $\Delta \xi=\xi_{s}$ has disappeared.

This allows for a cleaner interpretation of the notion of self-correlations, which are identified as correlations generated by a single point source. This intuitively constitutes a more accurate reflection of what the self-correlations are expected to represent, namely, correlations of a single fluctuation or fluid cell with itself and, therefore, gives confidence that these quantities are being identified and subtracted correctly.

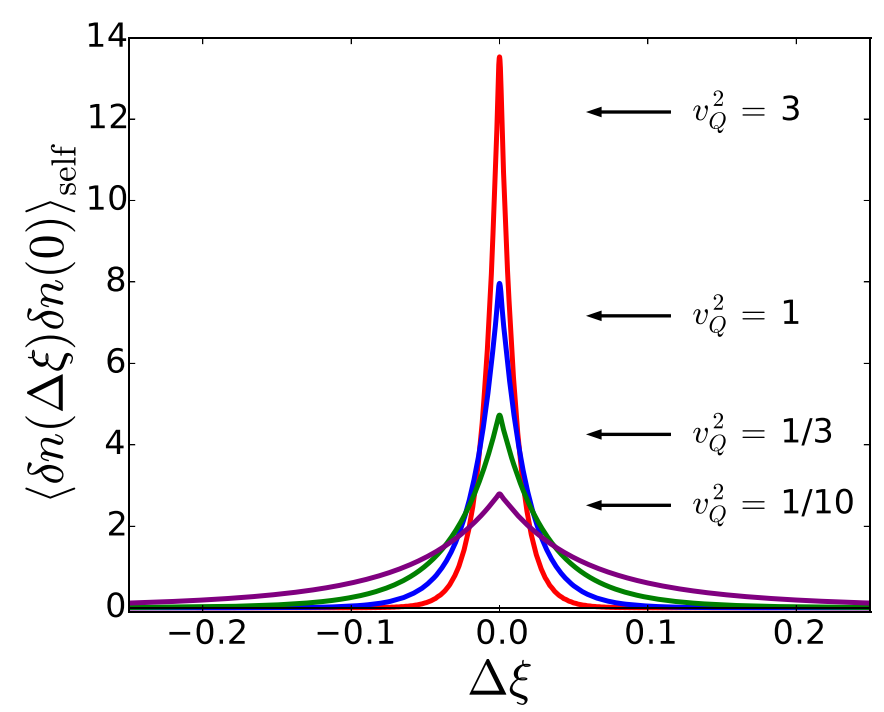

FIG. 1. The density-density self-correlation function versus $\Delta \xi$ for various values of $v_{Q}^{2}$ evaluated at the final time $\tau_{f}$. For large $v_{Q}^{2}$, it approaches a Dirac $\delta$ function. 


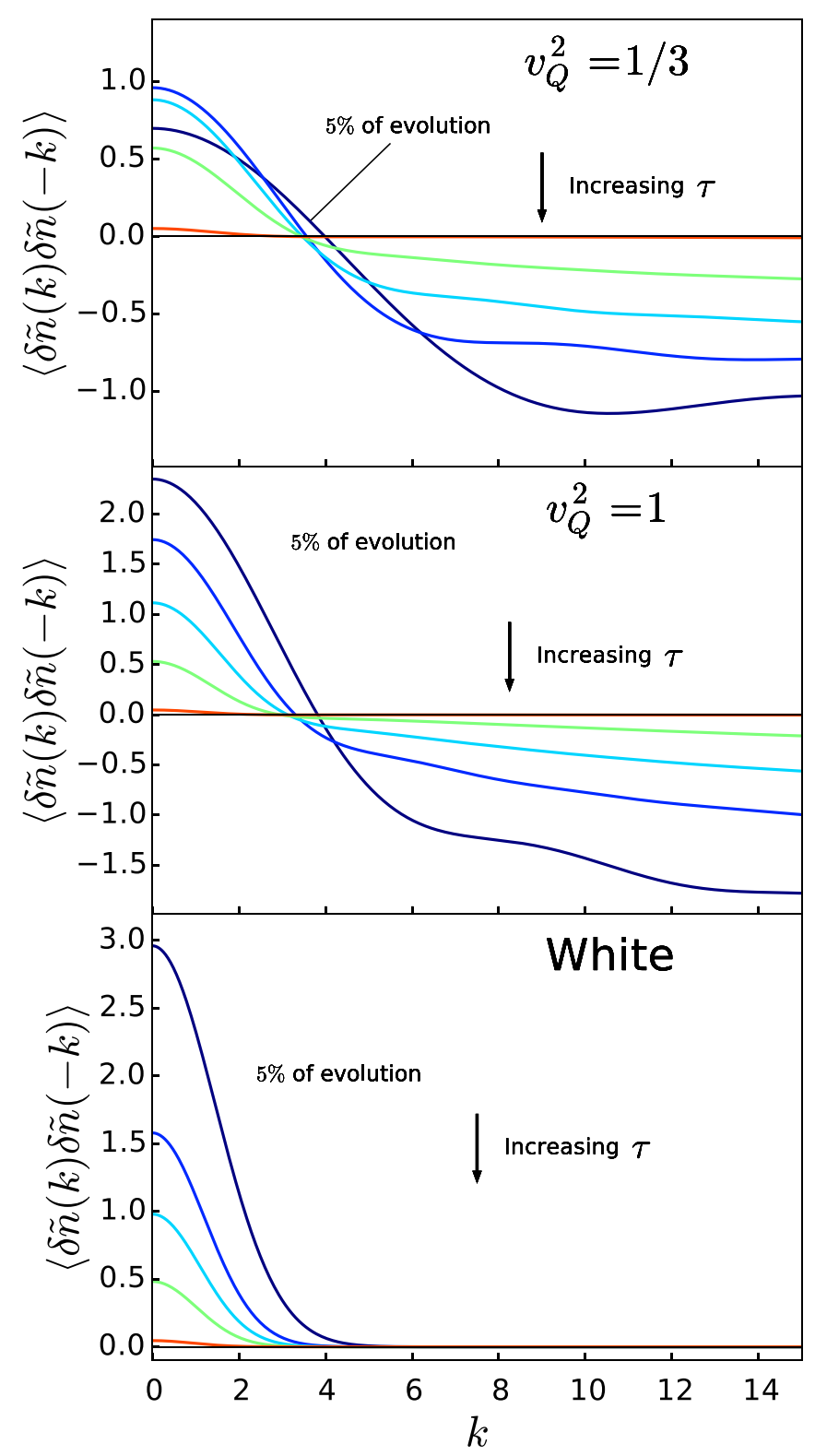

FIG. 2. Time evolution of the density-density correlation function (with self-correlations subtracted) versus $k$. Top panel: $v_{Q}^{2}=1 / 3$. Middle panel: $v_{Q}^{2}=1$. Bottom panel: ordinary diffusion with $v_{Q}^{2} \rightarrow \infty$. The different curves in each panel correspond to elapsed times of 5, 10, 15, 25 , and $100 \%$ of the system's lifetime $\tau_{f}-\tau_{0}=5.852 \mathrm{fm} / c$, starting at the top and working down at $k=0$. 

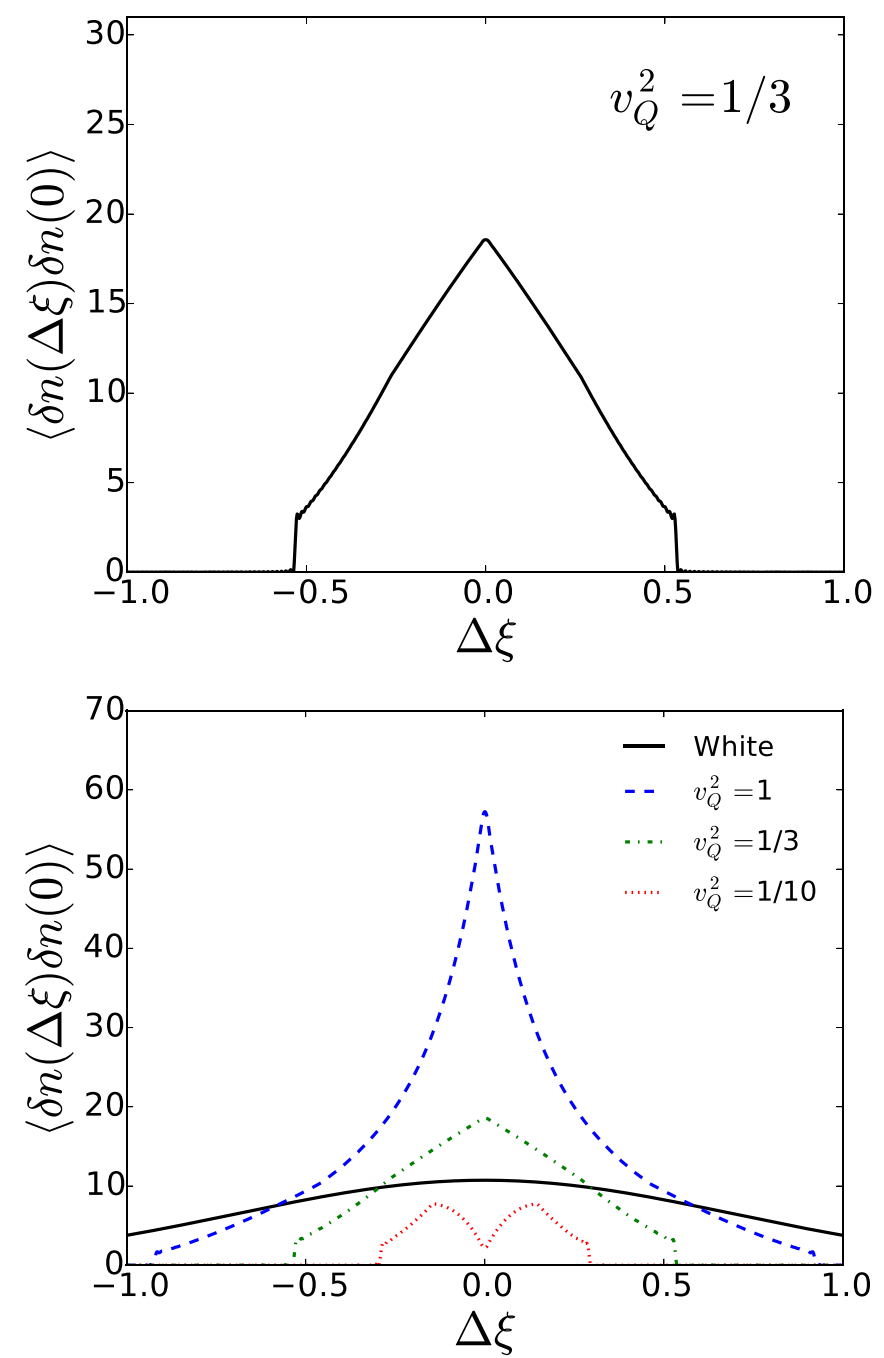

FIG. 3. The regular part of the density-density correlator in $\xi$ space after $5 \%$ of the total expansion time of $\tau_{f}-\tau_{0}=5.852$ fm $/ c$ has elapsed.

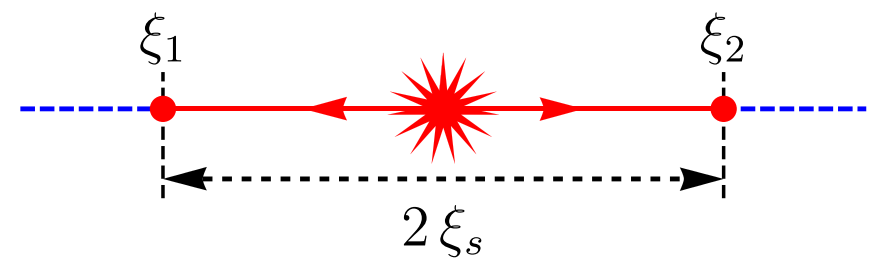

FIG. 4. Schematic of fluctuations and their horizons. 


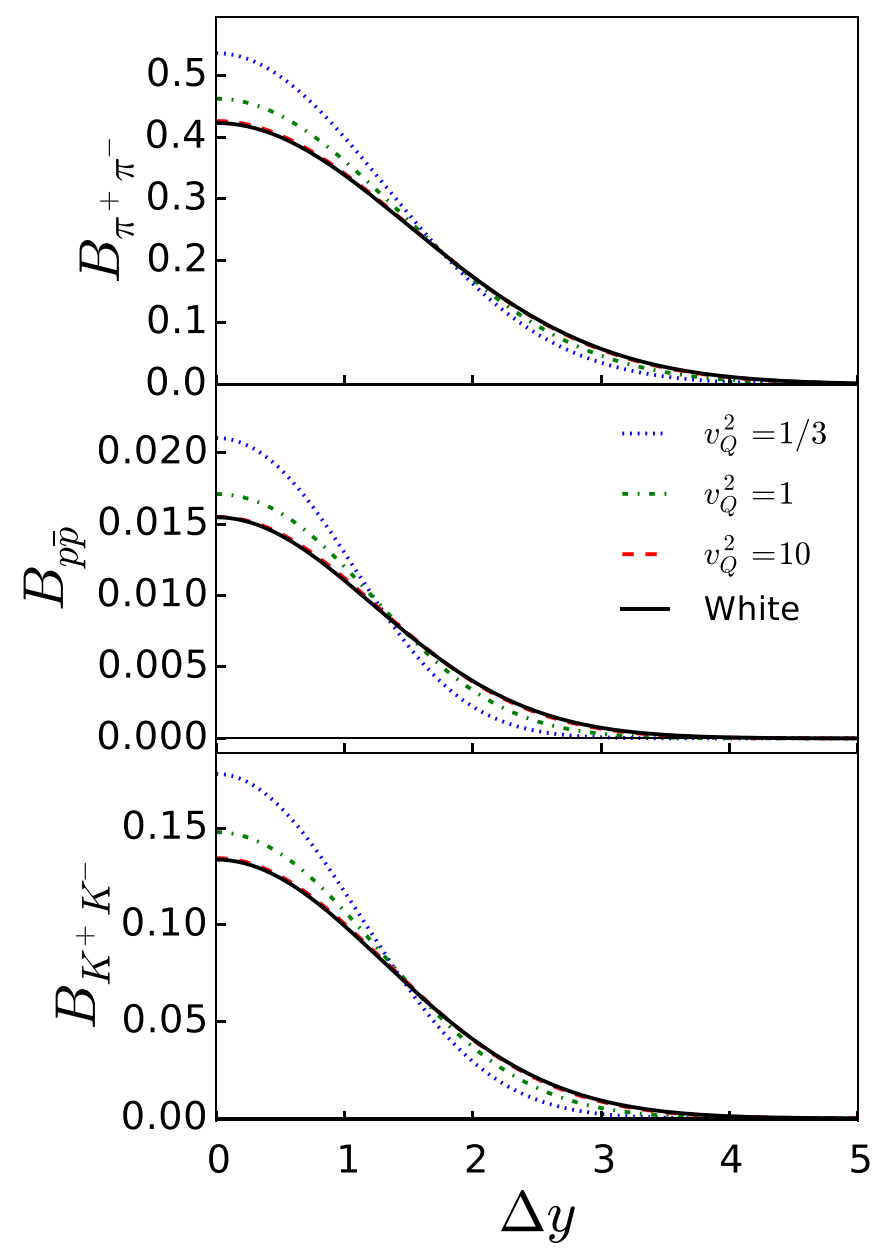

FIG. 5. Balance functions for pions, protons, and kaons. Looking at $\Delta y=0$, the curves correspond to $v_{Q}^{2}=1 / 3$ (top), $v_{Q}^{2}=1$ (middle), and $v_{Q}^{2}=10$ (bottom), with $v_{Q}^{2}=10$ being indistinguishable from the case of white noise in the ordinary diffusion equation.

This work was supported by the US DOE Grant No. DE-FG02-87ER40328. C.P. acknowledges support from the CLASH Project No. KAW 2017-0036.

[1] A. De, C. Plumberg, and J. I. Kapusta, arXiv:2003.04878. 